\title{
Aproveitamento do Óleo das Amêndoas de Tucumã do Amazonas na Produção de Biodiesel
}

\author{
Banny Silva BARBOSA ${ }^{1}$, Hector Henrique Ferreira KOOLEN ${ }^{2}$, Andreza Cruz BARRETO ${ }^{3}$, \\ João Domingos da SILVA ${ }^{4}$, Roberto FIGLIUOLO ${ }^{5}$, Sergio Massayoshi NUNOMURA ${ }^{6}$
}

\section{RESUMO}

A falta de disponibilidade de energia elétrica é um dos principais motivos pelo baixo Índice de Desenvolvimento Humano das comunidades isoladas localizadas na Amazônia. O biodiesel produzido a partir de óleos vegetais extraídos de espécies oleaginosas nativas de forma sustentada é uma das melhores alternativas energéticas para a região. O tucumã do amazonas, Astrocaryum aculeatum, é uma espécie de palmeira que produz um fruto muito apreciado na região, a partir do qual se obtém uma amêndoa com alto teor de óleo. Nesse estudo, foi avaliada a produção de biodiesel etílico, a partir de diferentes lotes de óleos de tucumã do amazonas, com índices de acidez baixos e elevados, pela transesterificação por catálise básica e ácida

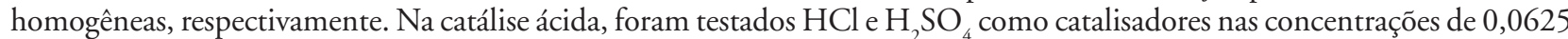
a $1,000 \mathrm{M}$, empregando etanol hidratado na proporção molar de $1: 6$ e a reação conduzida a $90{ }^{\circ} \mathrm{C}$ por $24 \mathrm{~h}$. Na catálise básica, foram testados $\mathrm{NaOH}$ e $\mathrm{KOH}$, nas proporçôes de 0,5 a 2,0 \%, empregando etanol anidro na proporção molar de 1:12 e a reação conduzida a $80^{\circ} \mathrm{C}$ por $2 \mathrm{~h}$. O biodiesel obtido em cada experimento foi analisado por métodos físicos (massa específica) e cromatográficos (CLAE em fase reversa). Análises cromatográficas indicaram que as melhores conversōes foram alcançadas por amostras de biodiesel com massas específicas inferiores a $0,87 \mathrm{~g} . \mathrm{cm}^{-1}$. As amostras de biodiesel obtidas com melhor qualidade foram obtidas utilizando-se os catalisadores ácidos a 1,0 M com rendimentos superiores a 90\%. No caso da catálise básica, obteve-se biodiesel de boa qualidade empregando-se o catalisador $\mathrm{NaOH}$ a 2,0\%, porém com rendimento inferior a $60 \%$. Contudo, em ambos os casos, foi possível identificar um excelente potencial de produção de biocombustível, a partir do óleo das amêndoas de tucumã.

PALAVRAS-CHAVE: Sustentabilidade, Arecaceae, Energia elétrica, Amazônia.

\section{The use of tucumã of amazonas kernel oil in the biodiesel production}

\begin{abstract}
The shortage of electricity is a major reason for the low Human Development Index of isolated communities located in the Amazon basin. The biodiesel produced from vegetable oils extracted from oil seeds native species, in a sustainable way, is one of the best alternative energy for the region. The "tucumã of amazonas", Astrocaryum aculeatum, is an Amazon palm tree that produces a much appreciated fruit in the region, from which it obtains kernels that have high content in oil. In this study, we evaluated the production of ethyl biodiesel, from different batches of oil obtained from "tucumã of amazonas" kernels with high and low acidity by the transesterification process acidic and basic catalysis, respectively. Different acid catalysts $(\mathrm{HCl}$ and $\mathrm{H}_{2} \mathrm{SO}_{4}$ ) and concentrations of each acid (from 0.0625 to $1.000 \mathrm{M}$ ) were tested, with ethanol at molar ratio of 1:6 at $90^{\circ} \mathrm{C}$ for $24 \mathrm{~h}$. In the basic catalysis, $\mathrm{NaOH}$ and $\mathrm{KOH}$ were tested at the concentration range of 0.5 to $2.0 \%$ with anhydrous ethanol with molar ratio $1: 12$ and the reactions took place at $80^{\circ} \mathrm{C}$ for $2 \mathrm{~h}$. The conversion into biodiesel and its quality was analyzed by means reversed-phase high performance liquid chromatography (RP-HPLC) and specific gravity. Chromatographic analysis indicated that the best conversions were achieved by samples of biodiesel with lowest specific gravity $\left(0.87 \mathrm{~g} . \mathrm{cm}^{-1}\right)$. The samples of biodiesel with best quality were obtained with acid catalysis at $1.0 \mathrm{M}$ with yield above $90 \%$. In the basic catalysis, it could be obtained biodiesel with good quality with $\mathrm{NaOH}$ at $2.0 \%$, but at lower yields. However in both types of catalysis, it was possible to identify an excellent potential for the production of this important biofuel from tucumã kernel oil.
\end{abstract}

KEY WORDS: Sustaintability, Arecaceae, Energy, Amazon.

\footnotetext{
1 Universidade Federal do Amazonas - banny_barbosa@yahoo.com.br

2 Universidade Federal do Amazonas - hector_ferreira86@yahoo.com.br

${ }^{3}$ Universidade Federal do Amazonas - andrezacb@yahoo.com.br

${ }^{4}$ Instituto Nacional de Pesquisas da Amazônia - jdomingo@inpa.gov.br

5 Instituto Nacional de Pesquisas da Amazônia - robfigli@inpa.gov.br

${ }^{6}$ Instituto Nacional de Pesquisas da Amazônia - smnunomu@inpa.gov.br
} 


\section{INTRODUÇÃO}

A região Amazônica, apesar de compreender mais de cinqüenta por cento do território brasileiro, possui problemas quase sempre dissociados da realidade do centro-sul do Brasil (Pereira Jr. et al., 2008). Um desses problemas é a falta de acesso à energia elétrica, especialmente nas comunidades isoladas. No Estado do Amazonas, em 2005, apenas 32 das mais de 4.600 comunidades isoladas $(0,7 \%)$ eram supridas por energia elétrica por meio da concessionária responsável (Correia, 2005).

A falta de acesso à energia elétrica é diretamente responsável pelo baixo índice de desenvolvimento humano das populaçōes residentes nas comunidades isoladas localizadas na Amazônia. O desafio do atendimento em energia elétrica no Brasil, em especial na Amazônia, é proporcional ao enfrentamento do alto nível desigual social e regional do país (Brasil, 2003).

A principal dificuldade para estender o acesso à energia elétrica na Amazônia é a grande dimensão territorial da região, fato esse que impede a extensão das redes de eletrificação como ocorre no restante do país (Bajay, 2005). A alternativa atualmente empregada para viabilizar a geração de energia elétrica nessas comunidades isoladas é a instalação de pequenos sistemas geradores elétricos a diesel. Contudo, do ponto de vista econômico, essa solução não é favorável, entre outros fatores, devido aos custos de transporte do combustível para essas comunidades isoladas (Correia, 2005).

O emprego de recursos naturais de forma manejada na geração local de energia elétrica vem sendo considerado uma das alternativas mais promissoras, em especial, o biodiesel, produzido a partir de óleos vegetais extraídos de espécies oleaginosas nativas. Óleos vegetais (triacilglicerídeos) podem ser convertidos, por exemplo, a partir de reaçōes de transesterificação com um álcool de cadeia curta (metanol ou etanol), na presença de um catalisador; numa mistura de ésteres de ácidos graxos (biodiesel) e glicerina (Schuchardt $e t$ al, 1998; Meher et al., 2006).

Portanto o biodiesel é uma energia alternativa e renovável, cujo uso apresenta importantes benefícios para o homem, como a diminuição da emissão dos gases que contribuem para o efeito estufa (ciclagem de carbono) e não emissão de compostos tóxicos contendo enxofre, nitrogênio ou poliaromáticos (Quadrelli \& Peterson, 2007; Bozbas, 2008).

Por outro lado, no contexto amazônico, a produção de biodiesel pode ser prejudicial ao homem, caso não leve em consideração a origem dos óleos vegetais. Estudos demonstram que a derrubada de florestas para o plantio de espécies oleaginosas domesticadas seria responsável por uma emissão muito maior de gases que contribuem para o efeito estufa, do que seria possível reduzir com o seu uso (Righelato \& Spracklen, 2007; Scharlemann \& Laurance, 2007).

Entretanto, a exploração manejada e sustentada de espécies oleaginosas nativas oriundas da biodiversidade Amazônica, especialmente aquelas que ocorrem em adensamentos florestais, é uma alternativa que concilia a geração de energia elétrica e o risco ambiental.

Dentre essas espécies oleaginosas nativas, destaca-se o tucumã do amazonas (Astrocaryum aculeatum G. Mey.) que é uma palmeira que pertence à família Arecacea, cujo mesocarpo é comestível e muito apreciado pela população do Estado do Amazonas (Lorenzi, 2004). O consumo vem gerando como resíduo o endocarpo, que contém uma amêndoa, a partir da qual se pode extrair óleo (Cavalcante, 1996).

Este estudo avaliou o emprego do óleo obtido das amêndoas do tucumã do amazonas, que até o momento não tem aproveitamento econômico, na produção de biodiesel pela via etílica.

\section{MATERIAIS E MÉTODOS}

\section{ÓLEOS}

Os óleos de tucumã do amazonas extraídos de amêndoas foram adquiridos em quatro diferentes lotes, o primeiro obtido por prensagem mecânica, enquanto os demais lotes foram obtidos pela extração sólido-líquido, com hexano, utilizando uma aparelhagem de Soxhlet. Os óleos obtidos por prensagem mecânica foram obtidos com rendimento médio de $20 \%$, enquanto que os obtidos por extração em Soxhlet de $40 \%$. Antes do uso, os óleos foram apenas colocados em rotaevaporador, sob pressão reduzida, a cerca de $50{ }^{\circ} \mathrm{C}$ para remover traços de solvente. Não foi realizado processo de refino algum para esses óleos.

\section{CARACTERIZAÇÃO FÍSICO-QUÍMICA DOS ÓLEOS}

As massas específicas tanto de óleo como de biodiesel foram determinadas em balóes volumétricos aferidos a $25^{\circ} \mathrm{C}$. Foram determinados os seguintes índices dos óleos disponíveis: índices de acidez (mg KOH/g óleo), saponificação (mg $\mathrm{NaOH} / \mathrm{g}$ de óleo), iodo (g $\mathrm{I}_{2} / 100 \mathrm{~g}$ óleo) e peróxidos (meq/ Kg de óleo), utilizando metodologias descritas por Moretto e Fett (1989), e pela Horwitz (2000). Todos esses procedimentos foram realizados em triplicata.

\section{DETERMINAÇÃO DA CADEIA DE ÁCIDOS GRAXOS POR CROMATOGRAFIA GASOSA (CG)}

A composição dos ácidos graxos presentes nos óleos foi determinada para definir o peso molecular médio dos óleos disponíveis. Os óleos (compostos preponderantemente por triacilglicerídeos) foram inicialmente convertidos nos seus respectivos ésteres metílicos, conforme método oficial AOAC 
969.33 (Horwitz, 2000). A composição da mistura de ésteres metílicos foi determinada por cromatografia gasosa de alta resolução nas seguintes condições. Cromatógrafo gasoso da marca Agilent, modelo HP 6890 Plus, equipado com sistema de aquisição e manipulação de dados (Chemstation versão A.10.02) e configurado com duas colunas capilares de dimensôes idênticas ( $30 \mathrm{~m}$ de comprimento, $0,32 \mathrm{~mm}$ de diâmetro interno e espessura do filme de $0,25 \mu \mathrm{m}$ ), em paralelo, uma coluna polar (HP INNOWAX-20) e outra apolar (HP-5), gás de arraste hidrogênio a 11,7 psi, sistema de injeção automático com volume de injeção de $1 \mu \mathrm{L}$, injetor no modo split com razão 20:1 e temperatura de 250 ${ }^{\circ} \mathrm{C}$, detetores de ionização de chama a $260{ }^{\circ} \mathrm{C}$. A temperatura inicial do forno foi de $120^{\circ} \mathrm{C}$ com uma rampa de $3^{\circ} \mathrm{C} / \mathrm{min}$ até a temperatura de $220^{\circ} \mathrm{C}$ e mantida nessa temperatura por 6 min. Os ésteres metílicos das amostras foram identificados com auxílio de amostras autênticas (Sigma-Aldrich e Carlo Erba). A quantificação foi realizada pelo método de normalização de área, considerando apenas os picos identificados.

ANÁLISES DE CONVERSÃO DE ÉSTERES ETÍLICOS POR CROMATOGRAFIA LÍQUIDA DE ALTA EFICIÊNCIA (CLAE)

As amostras de conversão do óleo em biodiesel foram realizadas por CLAE empregando o método descrito por Holcapeck (1999) com algumas modificações. As análises foram realizadas num cromatógrafo Shimadzu, modelo Prominence, equipado com bomba quaternária, modelo LC-10AVp, sistema de injeção automático, SIL-20A, detetor do tipo de arranjo de diodos, SPD-M20A, e software de manipulação Solution. Foi empregada uma coluna de fase reversa Merck Lichrospher 100 RP-18e de $5 \mu \mathrm{m}$ (250 x 4 $\mathrm{mm}$ ) e fluxo de $1,0 \mathrm{~mL} / \mathrm{min}$. Foi empregado o seguinte modo gradiente: 0 min $-\mathrm{H}_{2} \mathrm{O} / \mathrm{ACN}(30: 70) ; 2 \mathrm{~min}-\mathrm{H}_{2} \mathrm{O} / \mathrm{ACN}$ (30:70); $8 \mathrm{~min}-\mathrm{H}_{2} \mathrm{O} / \mathrm{ACN}(10: 90) ; 22 \mathrm{~min}-\mathrm{H}_{2} \mathrm{O} / \mathrm{ACN}$ (30:70); $27 \mathrm{~min}-\mathrm{ACN} / \mathrm{Hexano} / \mathrm{i}-\mathrm{PrOH}(30: 28: 42) ; 32 \mathrm{~min}$ ACN/Hexano/i-PrOH (30:28:42). Cada amostra de biodiesel foi diluída 50 vezes com uma mistura de hexano/i-PrOH (1:1) e injetados $40 \mu \mathrm{L}$. Foram injetados diferentes padrōes ésteres metílicos, ácidos graxos, mono, di e triacilglicerídeos (Sigma e Carlo Erba) para auxiliar na identificação desses componentes em cada biodiesel produzido. A conversão em ésteres etílicos (biodiesel) foi determinada pelo método de quantificação por normalização de área dos cromatogramas obtidos a $215 \mathrm{~nm}$.

\section{CONDIÇÕES DE REAÇÃO E CATALISADORES TESTADOS NA CATÁLISE ÁCIDA}

A catálise ácida foi empregada com o lote 1, com índice de acidez de $37,5 \mathrm{mg} \mathrm{KOH} / \mathrm{g}$ de óleo. As reaçôes foram realizadas empregando $60 \mathrm{~g}$ de óleo em balão de fundo redondo, em sistema de refluxo, sob aquecimento e agitação constante.

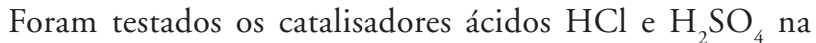
concentraçōes 0,0625 a 1,000 M (concentraçōes do catalisador na mistura reacional), empregando etanol hidratado na relação molar óleo/etanol $1: 6$, a $90^{\circ} \mathrm{C}$ por $24 \mathrm{~h}$.

\section{CONDIÇÕES DE REAÇÃO E CATALISADORES TESTADOS NA CATÁLISE BÁSICA}

O lote 3, com índice de acidez de 1,98 mg KOH/g, foi empregado para testar os catalisadores básicos $\mathrm{NaOH}$ e $\mathrm{KOH}$ nas concentrações de $0,5 \%$ a 2,0 \% (em peso), empregando uma relação molar óleo/etanol anidro de $1: 12,80^{\circ} \mathrm{C}$ e por $2 \mathrm{~h}$. Também foi realizado um experimento de conversão em biodiesel, utilizando $\mathrm{KOH}$ a 2,0 \% em quatro proporçōes molares óleo/etanol (entre 1:6 e 1:18), a $80^{\circ} \mathrm{C}$ e por $2 \mathrm{~h}$..

\section{TRATAMENTO PÓS-REACIONAL E AVALIAÇÃO DO BIODIESEL}

Ao final da reação, as fases foram separadas (no caso da via etílica, necessária a adição de glicerina) e a fase superior contendo o biodiesel sofreu duas lavagens, uma com solução neutralizadora $(\mathrm{NaOH}$ ou $\mathrm{HCl} 0,1 \mathrm{~N})$ e então com uma lavagem com água deionizada. Em seguida, a fase de biodiesel foi centrifugada à $15.557 \mathrm{~g}$ por 30 min para remoção da água residual. $\mathrm{O}$ volume final de biodiesel obtido foi medido e o rendimento obtido por pesagem. A qualidade do biodiesel foi determinada pela avaliação da massa específica, que também foi utilizada para indicar a conversão em ésteres etílicos. Froehner et al (2007) demonstraram a correlação linear entre a massa específica e o teor de éster obtido por cromatografia. A massa específica é uma das propriedades físicas, que serve de parâmetro de qualidade do biodiesel em vários países (Meher et al., 2006). Quanto menor a massa específica, maior é a conversão em ésteres e consequentemente melhor é a qualidade do biodiesel. Apesar da Resolução ANP 042 (DOU, 2004) não estabelecer um limite, convém ressaltar que outras normas estabelecem um intervalo, como é o caso da norma européia EM 14.214, que estabelece um intervalo de $0,86-0,89 \mathrm{~g} . \mathrm{cm}^{-3}$.

\section{RESULTADOS E DISCUSSÃO}

As caracterizações físicas e químicas foram realizadas para todos os quatro lotes, onde se obteve os seguintes resultados:

A principal diferença observada foi no índice de acidez, onde nos dois primeiros lotes ( 1 e 2), foram obtidos índices de acidez bastante elevados, o que inviabiliza a produção de biodiesel pela transesterificação por catálise básica homogênea, que é o processo mais largamente utilizado na produção de biodiesel, para esses dois lotes. Nos dois últimos lotes (3 e 4), os índices de acidez foram bem inferiores, tornando possível a produção de biodiesel pela transesterificação por catálise básica homogênea.

Os demais índices indicaram que o óleo das amêndoas é composto de resíduos de ácidos graxos saturados (baixos 
Tabela 1 - Propriedades e índices físico-químicos dos quatro lotes de óleos de amêndoas

\begin{tabular}{lccccc}
\hline Lote & $\begin{array}{c}\text { Índice de Acidez } \\
(\mathrm{mg} \mathrm{KOH} / \mathrm{g})\end{array}$ & $\begin{array}{c}\text { Índice de Saponificação } \\
(\mathrm{mg} \mathrm{NaOH} / \mathrm{g})\end{array}$ & $\begin{array}{c}\text { Índice de Peróxidos } \\
\left(\mathrm{meq} \mathrm{O}_{2} / \mathrm{kg}\right)\end{array}$ & $\begin{array}{c}\text { Índice de lodo } \\
(\mathrm{g} \mathrm{l} / 100 \mathrm{~g})\end{array}$ & $\begin{array}{c}\text { massa específica } \\
\left(\mathrm{g} / \mathrm{cm}^{3}\right)\end{array}$ \\
\hline 1 & $37,5 \pm 0,4$ & $210,5 \pm 0,4$ & $13,9 \pm 0,9$ & $2,2 \pm 0,2$ & $0,8889 \pm 0,0089$ \\
2 & $79,4 \pm 0,2$ & $245 \pm 1$ & $1,4 \pm 0,1$ & $4,1 \pm 0,8$ & $0,9064 \pm 0,0016$ \\
3 & $1,98 \pm 0,01$ & $216 \pm 2$ & $3,6 \pm 0,6$ & $0,40 \pm 0,04$ & $0,8927 \pm 0,0005$ \\
4 & $5,0 \pm 0,1$ & $218,6 \pm 0,7$ & $5,0 \pm 0,2$ & $8,3 \pm 0,3$ & $0,8933 \pm 0,0017$ \\
\hline
\end{tabular}

Lote 1 obtido por prensagem mecânica e lotes 2 a 4 por Soxhlet com hexano

índices de iodo e peróxidos) e de cadeia curta (alto índice de saponificação).

As análises por cromatografia gasosa confirmaram esses resultados. Os ésteres metílicos das amostras dos quatro lotes mostraram que todos apresentavam cadeias de ácidos graxos, curtas e saturadas. A análise foi efetuada em duas colunas cromatográficas para uma maior confiabilidade dos resultados.

A composição percentual foi determinada pela média dos percentuais de áreas obtidos para cada um dos ésteres metílicos de ácidos graxos identificados nos cromatogramas obtidos em coluna polar e apolar. Foi possível identificar mais de $97 \%$ dos ésteres metílicos. De acordo com os resultados e apresentados na tabela abaixo, foi possível estimar o massa molecular média do óleo como sendo de 672,63 g/mol.

Por causa dos resultados dos índices de acidez obtidos, foi avaliada a síntese de biodiesel por dois diferentes tipos de catálise, ácida e básica, empregando catalisadores homogêneos mais comumente empregados.

\section{CATÁLISE ÁCIDA}

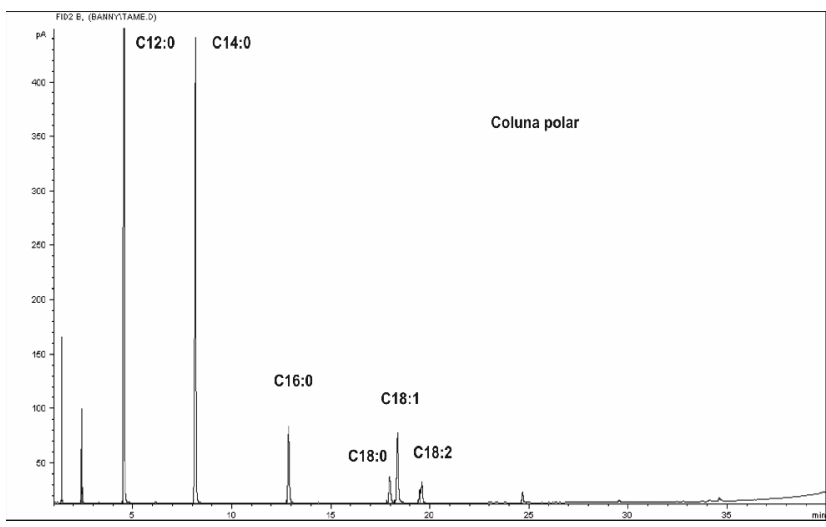

Tabela 2 - Composição da cadeia graxa do óleo de tucumã obtido por cromatografia gasosa.

\begin{tabular}{lccc}
\hline Éster metílico & \% Área $_{\text {(coluna apolar) }}$ & \% Área $_{\text {(coluna polar) }}$ & \% Área \\
\hline C8́dia \\
\hline C 10:0 & 2,04 & 2,03 & 2,03 \\
C 12:0 & 1,88 & 1,79 & 1,83 \\
C 14:0 & 51,74 & 51,10 & 51,42 \\
C 16:0 & 26,17 & 26,01 & 26,09 \\
C 18:0 & 5,55 & 5,54 & 5,55 \\
C 18:1 $(n=9)$ & 2,36 & 2,36 & 2,36 \\
C 18:2 $(n=9)$ & 6,00 & 5,93 & 5,97 \\
$\Sigma$ & 2,15 & 2,04 & 2,09 \\
\hline
\end{tabular}

A transesterificação pela catálise ácida utilizou etanol hidratado e foram testados os catalisadores $\mathrm{HCl}$ e $\mathrm{H}_{2} \mathrm{SO}_{4}$. Foram avaliadas diferentes concentrações de catalisador e os resultados de rendimento e massa específica são apresentados na figura 1. A conversão do óleo em biodiesel pode ser observada claramente pela variação da massa específica, cujo resultado foi confirmado por CLAE (tabela 3).

Pela figura 2 e tabela 3, um resultado semelhante pode ser observado para ambos os catalisadores ácidos com uma boa conversão do óleo em biodiesel. Ocorreu uma leve redução de rendimento para o ácido sulfúrico nas concentraçôes mais

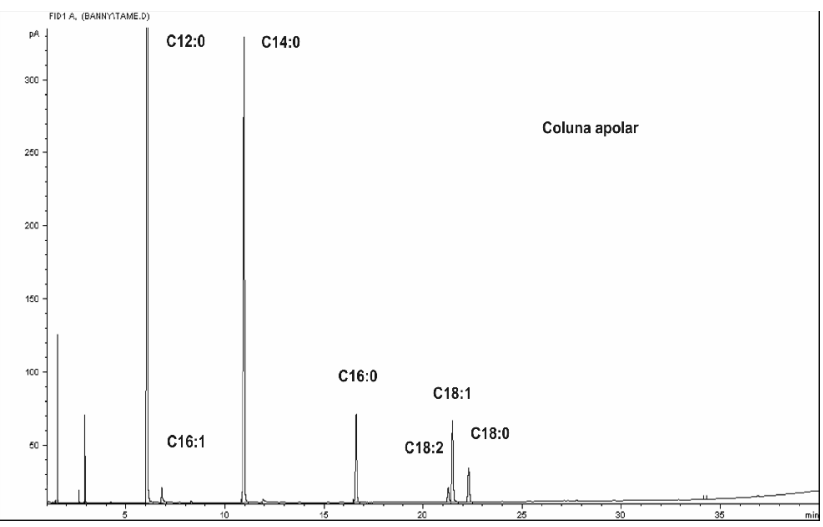

Figura 1 - Cromatogramas dos ésteres metílicos do óleo do lote 1, obtidos por cromatografia gasosa de alta resolução em colunas polar e apolar. 


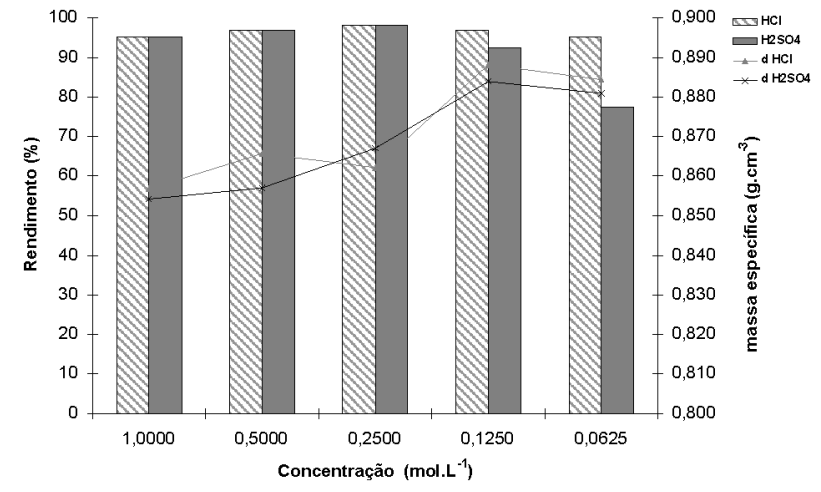

Figura 2 - Rendimento e massa específica de biodiesel obtido usando como catalisadores $\mathrm{HCl}$ e $\mathrm{H}_{2} \mathrm{SO}_{4}$

baixas. Vale ressaltar que as análises por CLAE e de massa específica, indicaram que houve uma melhor conversão, resultando numa boa qualidade dos combustíveis obtidos, nas concentrações superiores a $0,25 \mathrm{~mol} \cdot \mathrm{L}^{-1}$, para ambos os catalisadores.

\section{CATÁLISE BÁSICA}

Foi empregada a transesterificação por catálise básica homogênea, onde foram testados os catalisadores $\mathrm{NaOH}$ e $\mathrm{KOH}$. Os resultados obtidos são apresentados na figura 3 .

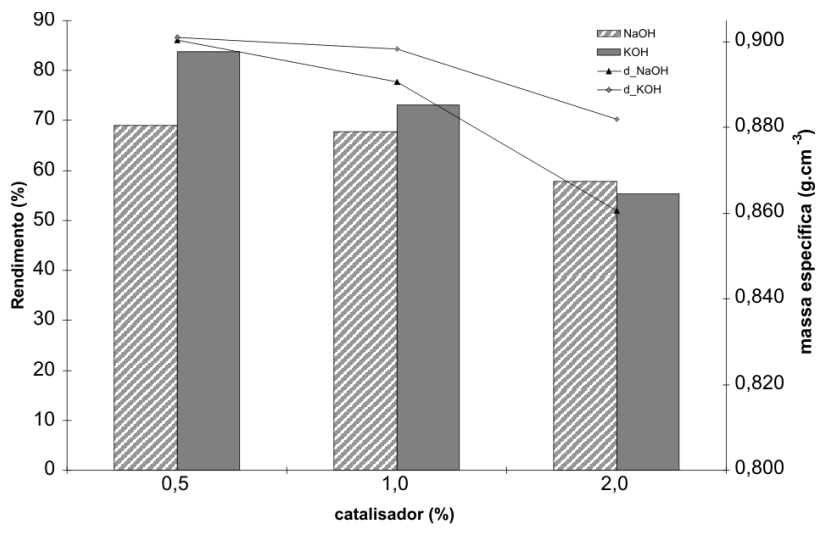

Figura 3 - Rendimento e massa específica de biodiesel etílico obtido usando como catalisadores $\mathrm{NaOH}$ e KOH

$\mathrm{Na}$ figura 3, é possível observar que utilizando 0,5 a $1,0 \%$ em peso do catalisador, o $\mathrm{KOH}$ proporcionou melhor rendimento de biodiesel etílico, se comparado ao $\mathrm{NaOH}$. Os menores valores de massa específica indicaram que a conversão com $\mathrm{NaOH}$ foi um pouco mais eficiente, resultados esses confirmados por CLAE (tabela 3). Contudo os menores rendimentos de biodiesel foram obtidos com o uso de $2 \%$ de catalisador.
Com base nesses resultados, avaliou-se ainda o emprego de $\mathrm{KOH}$ a $1 \%$ em massa como catalisador, na presença de diferentes concentrações de etanol anidro. As reaçôes foram realizadas a $80^{\circ} \mathrm{C}$ por $2 \mathrm{~h}$. Os resultados de rendimento e massa específica são apresentados na figura 4 .

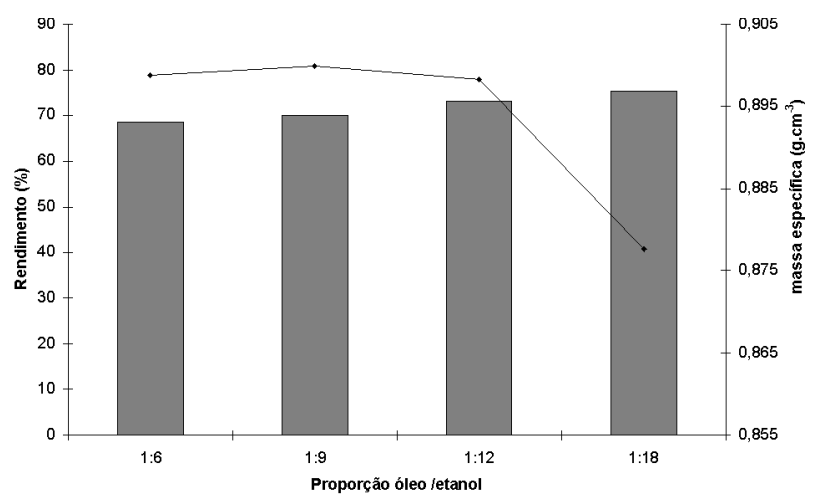

Figura 4 - Rendimento e massa específica de biodiesel etílico obtido a diferentes proporções molares óleo/álcool e 1\% de KOH

Observa-se que o aumento da proporção molar de etanol anidro, aumenta tanto o rendimento da reação, quanto o percentual de conversão, observado pela redução da massa específica do biodiesel produzido.

Tabela 3 - Resultados da análise da conversão em ésteres etílicos (biodiesel) por cromatografia líquida de alta eficiência.

\begin{tabular}{llllll}
\hline $\begin{array}{l}\text { Catalisador } \\
\text { (relação } \\
\text { molar) }\end{array}$ & $\begin{array}{l}\text { Quantidade } \\
\text { catalisador }\end{array}$ & $\begin{array}{l}\text { Teor de } \\
\text { éster } \\
(\%)\end{array}$ & $\begin{array}{l}\text { Catalisador } \\
\text { (relação } \\
\text { molar) }\end{array}$ & $\begin{array}{l}\text { Quantidade } \\
\text { catalisador }\end{array}$ & $\begin{array}{l}\text { Teor de } \\
\text { éster (\%) }\end{array}$ \\
\hline $\mathrm{HCl}(1: 6)$ & $1,0000 \mathrm{M}$ & 76,3 & $\mathrm{H}_{2} \mathrm{SO}_{4}(1: 6)$ & $1,0000 \mathrm{M}$ & 72,1 \\
$\mathrm{HCl}(1: 6)$ & $0,5000 \mathrm{M}$ & 72,4 & $\mathrm{H}_{2} \mathrm{SO}_{4}(1: 6)$ & $0,5000 \mathrm{M}$ & 70,0 \\
$\mathrm{HCl}(1: 6)$ & $0,2500 \mathrm{M}$ & 72,9 & $\mathrm{H}_{2} \mathrm{SO}_{4}(1: 6)$ & $0,2500 \mathrm{M}$ & 68,6 \\
$\mathrm{HCl}(1: 6)$ & $0,1250 \mathrm{M}$ & 46,2 & $\mathrm{H}_{2} \mathrm{SO}_{4}(1: 6)$ & $0,1250 \mathrm{M}$ & 49,2 \\
$\mathrm{HCl} \mathrm{(1:6)}$ & $0,0625 \mathrm{M}$ & 47,0 & $\mathrm{H}_{2} \mathrm{SO}_{4}(1: 6)$ & $0,0625 \mathrm{M}$ & 46,5 \\
$\mathrm{NaOH}(1: 12)$ & $2,00 \%$ & 56,0 & $\mathrm{KOH} \mathrm{(1:12)}$ & $2,00 \%$ & 43,9 \\
$\mathrm{NaOH}(1: 12)$ & $1,00 \%$ & 40,6 & $\mathrm{KOH} \mathrm{(1:12)}$ & $1,00 \%$ & 29,0 \\
$\mathrm{NaOH} \mathrm{(1:12)}$ & $0,50 \%$ & 32,8 & $\mathrm{KOH} \mathrm{(1:12)}$ & $0,50 \%$ & 21,8 \\
$\mathrm{KOH}(1: 6)$ & $2,00 \%$ & 31,1 & $\mathrm{KOH} \mathrm{(1:12)}$ & $2,00 \%$ & 29,0 \\
$\mathrm{KOH}(1: 9)$ & $2,00 \%$ & 38,5 & $\mathrm{KOH} \mathrm{(1:18)}$ & $2,00 \%$ & 32,5 \\
\hline
\end{tabular}

Entretanto, os rendimentos observados para a catálise básica (65 a 84\%) foram ainda bastante inferiores àqueles observados para a catálise ácida (76 a 95\%) e indicam uma necessidade de melhoria nas condiçôes reacionais para o emprego da via etílica na produção de biodiesel de óleo de amêndoas do tucumã do amazonas. 


\section{CONCLUSÃO}

$\mathrm{Na}$ execução desse trabalho, verificou-se que os óleos obtidos de amêndoas do tucumã do amazonas possuem preponderantemente cadeias hidrocarbônicas saturadas e curtas, podendo ter tanto índices de acidez relativamente baixos como altos. Contudo foi possível demonstrar a viabilidade da produção de biodiesel pela via etílica tanto para os óleos com índices de acidez mais baixos como mais elevados. Ainda são necessários estudos para melhorar a eficiência energética para os dois tipos de catálise, especialmente para reduzir os custos de produção (tempo de reação, temperatura de reação e diminuição de corrosão com o emprego de catalisadores ácidos não corrosivos), especialmente para viabilizar o emprego desse óleo na produção de biodiesel etílico em maior escala. Os baixos valores de conversão em ésteres etílicos observados especialmente na catálise básica homogênea podem ter sido causados pelo fato do óleo não ter sido submetido a processo algum de refino.

\section{AGRADECIMENTOS}

Ao CNPq pelas bolsas concedidas à $\mathrm{BSB}$ (IC) e SMN (DT). À FAPEAM pelas bolsas concedidas à ACB (MS). Esse projeto teve suporte financeiro da FAPEAM, FINEP e CNPq.

\section{BIBLIOGRAFIA CITADA}

Brasil 2003. Programa Nacional de Universalização do Acesso e Uso da Energia Elétrica. Manual de Operacionalização. Ministério de Minas e Energia. 30pp.

Bajay, S.V. 2005. Programa Nacional de Universalização do Acesso e Uso da Energia Elétrica. T\&C Amazônia, 6: 2-4.

Bozbas, K. 2008. Biodiesel as an alternative motor fuel: production and policies in the European Union. Renewable and Sustainable Energy Reviews, 12(2): 542-552.

Correia, J.C. 2005. Atendimento energético a pequenas comunidades isoladas: barreiras e possibilidades. T\&C Amazônia, 6: 30-35.

Cavalcante, P.B. 1996. Frutas comestiveis da Amazônia; coleção Adolfo Ducke, 6a edição, Belém Pará. p. 219-220.

DOU, 2004. Ministério das Minas e Energia. Agência Nacional do Petróleo. Resolução n. 42 de 24 de novembro de 2004. Edição N. 236 de 09/12/2004.
Froehner, S.; Leithold, J.; Lima Jr.; L.F. 2007. Transesterificação de óleos vegetais: caracterização por cromatografia em camada delgada. Química Nova, 30(8): 2016-2019.

Holcapeck, M., Jandera, P., Fischer, J., Prokes, B. 1999. Analytical monitoring of the production of biodiesel by high-performance liquid chromatography with various detection methods. Journal of Chromatography $A$, 858: 13-31.

Horwitz, W. 2000. Official Methods of Analysis of AOAC International. AOAC International, Maryland, EUA, 17 edição, volume II, capítulo 41, p. 1-69.

Lorenzi, H., Souza, H.M., Cerqueira; L.S.C.; Costa, J.T.M.; Ferreira, E. 2004. Palmeiras Brasileiras e exóticas cultivadas. Instituto Plantarum, Nova Odessa, SP, p. 44.

Meher, L.C.; Sagar, D.V.; Naik, S.N. 2006. Technical aspects of biodiesel production by transesterification - a review. Renewable and Sustainable Energy Reviews, 10: 248-268.

Moretto, E.; Fett, R. 1989. Óleos e gorduras vegetais (Processamento de análises). UFSC, Florianópolis, SC. p 133, 141, 144-145.

Pereira Jr., A.O., Soares, J.B., Oliveira, R.G., Queiroz, R.P. 2007. Energy in Brazil: Toward sustainable development? Energy Policy, 36(1): 73-83.

Quadrelli, R., Peterson, S. 2007. The energy-climate challenge: recent trends in $\mathrm{CO}_{2}$ emissions from fuel combustion. Energy Policy, 35(11): 5938-5952.

Righelato, R., Spracklen, D.V. 2007. Carbon mitigation by biofuels or by saving and restoring forests? Science, 317(5840): 902.

Scharlemann, J.P.W., Laurance, W.F. 2008. How green are biofuels? Science, 319(5859): 43-44.

Schuchardt, U.; Sercheli, R.; Vargas, R. 1998. Transesterification of Vegetable Oils: a review. Journal of the Brazilian Chemical Society, 9(1): 199-210.

Recebido em 15/02/2008

Aceito em 30/10/2008 